\pm 1 vs $-20 \% \pm 2, p=0.02)$. There were significant negative correlations between CFVR and basal septal $(r=-0.7$, $\mathrm{p}=0.003)$, mid-septal $(\mathrm{r}=-0.5, \mathrm{p}=0.037)$ and global $\mathrm{T} 1$ times $(r=-0.6, p=0.012)$ - figure 1 . Basal septal $\mathrm{T} 1$ times were significantly elevated in subjects with CMD - median $1309 \mathrm{~ms}$ (IQR 1301-1313) vs $1292 \mathrm{~ms}$ (IQR 1281-1295), $\mathrm{p}=0.028$. There was a trend towards increased mid-septal and global T1 times in the CMD group - figure 2 and table 2. There was no difference in $\mathrm{T} 2$ times between the groups.

Conclusions This is the first study to show that, similar to HCM, increased myocardial fibrosis is associated with reduced CFVR in ESRD. Although causation cannot be demonstrated in this study, it raises the fascinating question of whether myocardial fibrosis is 'the chicken or the egg' in this inverse relationship. Further mechanistic studies are needed to confirm this association and to determine which is the primary pathology. It is likely that the interplay between myocardial fibrosis and CMD contributes to the significant cardiac mortality seen in ESRD.

Conflict of Interest None to declare

\section{THE IMPACT OF EXERCISE AND DIET INTERVENTION ON LEFT ATRIAL FUNCTION IN TYPE 2 DIABETES: RESULTS FROM A RANDOMISED STUDY}

${ }^{1}$ Aseel Alfuhied, ${ }^{1}$ Gaurav Gulsin ${ }^{1} \mathrm{Emer}$ Brady, ${ }^{2}$ Kelly S PaKe, 'Lavaya Athan, ${ }^{2}$ Joseph Henson, ${ }^{2}$ Emma Redman, ${ }^{3}$ Thomas Yates, ${ }^{3}$ Melanie Davies, ${ }^{1}$ Anna-Marie Marsh, ${ }^{1}$ Gerry McCann, 'Anvesha Singh. 'University of Leicester, Leicester, UK; ${ }^{2}$ NIHR Leicester Biomedical Research Centre, University of Leicester; ${ }^{3}$ University Hospitals of Leicester NHS Trust

\subsection{6/heartjnl-2021-BCS.157}

Introduction The effects of low-energy diet or exercise on cardiovascular function in younger adults with type 2 diabetes (DIASTOLIC) study showed a significant improvement in left ventricle peak early diastolic strain rate in response to a 12 week programme of aerobic exercise in patients with type-2 diabetes (T2D). The impact of exercise and a low-calorie meal replacement plan (MRP) on left atrial (LA) function in T2D has not yet been explored. We investigated the effect of lifestyle intervention on LA volumetric and strain parameters by cardiac magnetic resonance (CMR) imaging.

Methods The DIASTOLIC study was a prospective, randomised, open-label, blind endpoint trial. Obese participants with T2D (aged 18-65 years) were randomized to a 12-week intervention of: aerobic exercise training or low energy $(\approx 810 \mathrm{kcal} /$ day) MRP. CMR was performed at baseline and week-12. Images were analysed using Medis v3.1. LA strain and strain rate (LAS/SR) were assessed using Feature Tracking (QStrain v2.0), corresponding to LA reservoir (LAS/SR-r), conduit (LAS/ SR-cd), and booster pump (LAS/SR-bp) using 4- and 2-chamber standard steady-state free precession cine images, and average values calculated. LA volumes (LAV) were measured on 4 a d 2-chamber cine images and LA emptying fraction (LAEF) was calculated using biplane area-length method (QMass v8.1) for total, passive and active EF.

Results 45 participants with T2D completed the trial and had analysable LA cine images (22 exercise and $23 \mathrm{MRP}$ ). There were no significant changes in the standard assessment of LA volumetric function (LAV/LAEF) measured on CMR or in LV filling pressure (E\e') measured on echocardiography, in either group. In the MRP group, there were significant reductions in BMI (4.8 kg/m2), mean systolic blood pressure (SBP)
$(13 \mathrm{mmHg})$, and a significant increase in LAS-r and LAS-bp $(29.9 \pm 7.0$ to $32.3 \pm 7.0, \mathrm{p}=0.036$ and $14.6 \pm 5.3$ to $17.2 \pm 3.7$, $\mathrm{p}=0.034$ ) (see Table). The exercise arm showed a small reduction in BMI $(0.8 \mathrm{~kg} / \mathrm{m} 2)$, no significant change in BP and a significant improvement in LASR-bp $(-1.19 \pm 0.3$ to $-1.32 \pm 0.4$; $\mathrm{p}=0.041)$.

Conclusion A low-calorie MRP led to significant weight loss and improved SBP, with associated improvement in LA reservoir and contractile function on CMR strain assessment. LAS could detect early LA reverse remodelling post-lifestyle intervention in young adults with T2D despite no change in volumetric measurements.

Conflict of Interest None

\section{18F-SODIUM FLUORIDE POSITRON EMISSION TOMOGRAPHY IN ACUTE AORTIC SYNDROME}

${ }^{1}$ Maaz Syed, ${ }^{2}$ Alexander Fletcher, ${ }^{2}$ Samuel Debono, ${ }^{1}$ Rachael Forsythe, ${ }^{2}$ Michelle Williams, ${ }^{3}$ Marc Dweck, ${ }^{1}$ Adriana Tavares, ${ }^{1}$ Mark Macaskill, ${ }^{1}$ Anoop Shah, ${ }^{1}$ Martin Denvir, ${ }^{4}$ Kelvin Lim, ${ }^{4}$ William Wallace, ${ }^{1}$ Jakub Kaczynski, ${ }^{1}$ Tim Clark, ${ }^{5}$ Stephanie Sellers, ${ }^{4}$ Neil Masson, ${ }^{4}$ Orwa Falah, ${ }^{4}$ Roderick Chalmers, ${ }^{4}$ Andrew Tambyraja, ${ }^{2}$ Edwin van Beek, ${ }^{2}$ David Newby. ${ }^{1}$ BHF Department for Cardiovascular Sciences, University of Edinburgh, Edinburgh, UK; ${ }^{2}$ Centre for Cardiovascular Sciences, University of Edinburgh; ${ }^{3}$ University of Edinburgh; ${ }^{4} \mathrm{NHS}$ Lothian; ${ }^{5}$ Centre for Heart Lung Innovation, University of British Columbia

\subsection{6/heartjnl-2021-BCS.158}

Background Acute aortic syndrome is a catastrophic condition characterised by medial degeneration and cellular destruction within the aortic wall. 18F-Sodium fluoride (18F-NaF) positron emission tomography (PET) detects microscopic calcification as a marker of disease activity. This proof-of-concept study aims characterise $18 \mathrm{~F}-\mathrm{NaF}$ PET in patients with acute aortic syndrome.

Methods Aortic tissue obtained from patients with acute aortic syndrome was stained using von Kossa's stain for calciumphosphate complexes and then exposed to $18 \mathrm{~F}$-sodium fluoride to confirm radiotracer binding to microcalcification. Next, patients with aortic dissection or intramural haematomas and healthy controls underwent 18F-NaF PET/CT and CT angiography of the aorta. A threshold of 12 weeks since diagnosis was used to classify patients to 'recent' or 'prior' acute aortic syndrome groups. Peak aortic $18 \mathrm{~F}-\mathrm{NaF}$ uptake was corrected for background blood pool activity to obtain a most-diseased segment tissue-to-background ratio (MDS TBRmax). Radiotracer binding was compared with aortic size in a linear regression model and major adverse aortic events (aortic rupture, aorta-related death or aortic repair) in a proportional hazards Cox survival analysis.

Results Aortic 18F-NaF uptake co-localized with histologically defined regions of microcalcification $(n=15)$. Patients with acute aortic syndrome had increased $18 \mathrm{~F}-\mathrm{NaF}$ binding compared to healthy controls (TBRmax 2.02 $\pm 0.42 \quad(n=47)$ vs $1.36 \pm 0.39(\mathrm{n}=20)$ respectively, $\mathrm{p}<0.001)$. Peak radiotracer uptake occurred at the site of intimal disruption $(+27.5 \%$ compared to the proximal aorta, $\mathrm{p}<0.001)$. $18 \mathrm{~F}-\mathrm{NaF}$ binding to the false lumen was associated with aortic growth $(+7.1$ $\mathrm{mm} / \mathrm{yr}, \mathrm{p}=0.011$ ) and uptake in the outer aortic wall was associated with major adverse aortic events (hazard ratio 8.6 [95\% CI, 1.1-68.1], $\mathrm{p}=0.041$ ) in patients with recent acute aortic syndrome.

Conclusion 18F-NaF PET/CT uptake was increased in patients with acute aortic syndrome at sites of disease activity. Radiotracer binding was associated with aortic growth and clinical 